\title{
Dual Cherenkov Radiation-induced Near-Infrared Luminescence Imaging and Photodynamic Therapy towards Tumor Resection
}

Vivian Lioret, $†$ Pierre-Simon Bellaye $\ddagger$ Christine Arnould, \# Bertrand Collin, $\ddagger$ Richard A. Decréau †*

† Université de Bourgogne Franche Comté, ICMUB Institute (Chemistry Department), Sciences

Mirande, 9 Avenue Alain Savary, 21078 Dijon, France

‡ Centre George François Leclerc, 1 rue du Professeur Marion, 21079 Dijon, France

\# INRA Dijon, Bâtiment CMSE, 17 rue Sully, Dijon, France

\section{Supporting Information}

Table of Contents

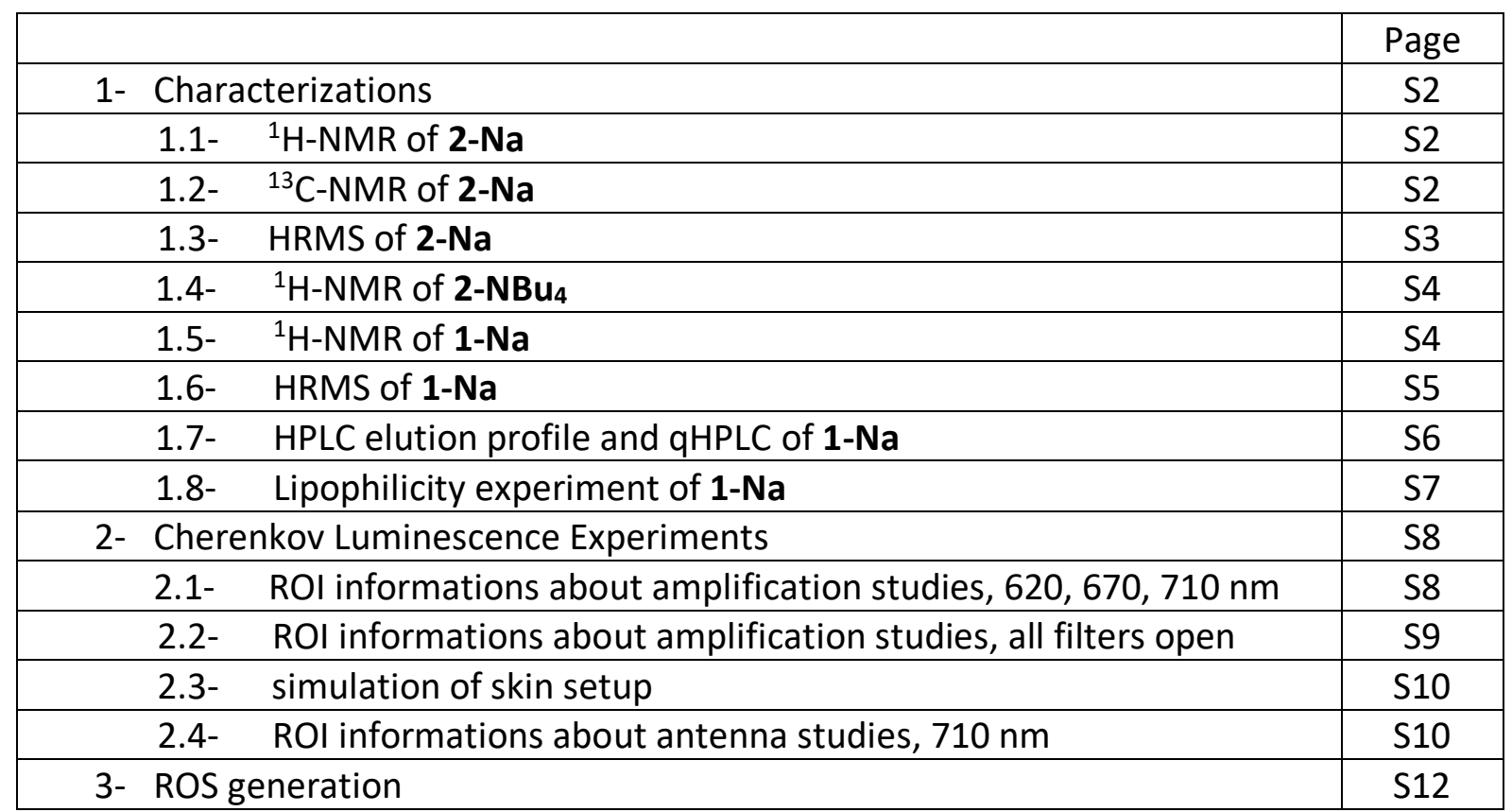




\section{1- Characterizations}

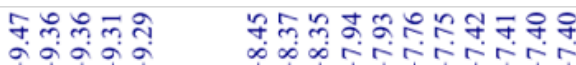
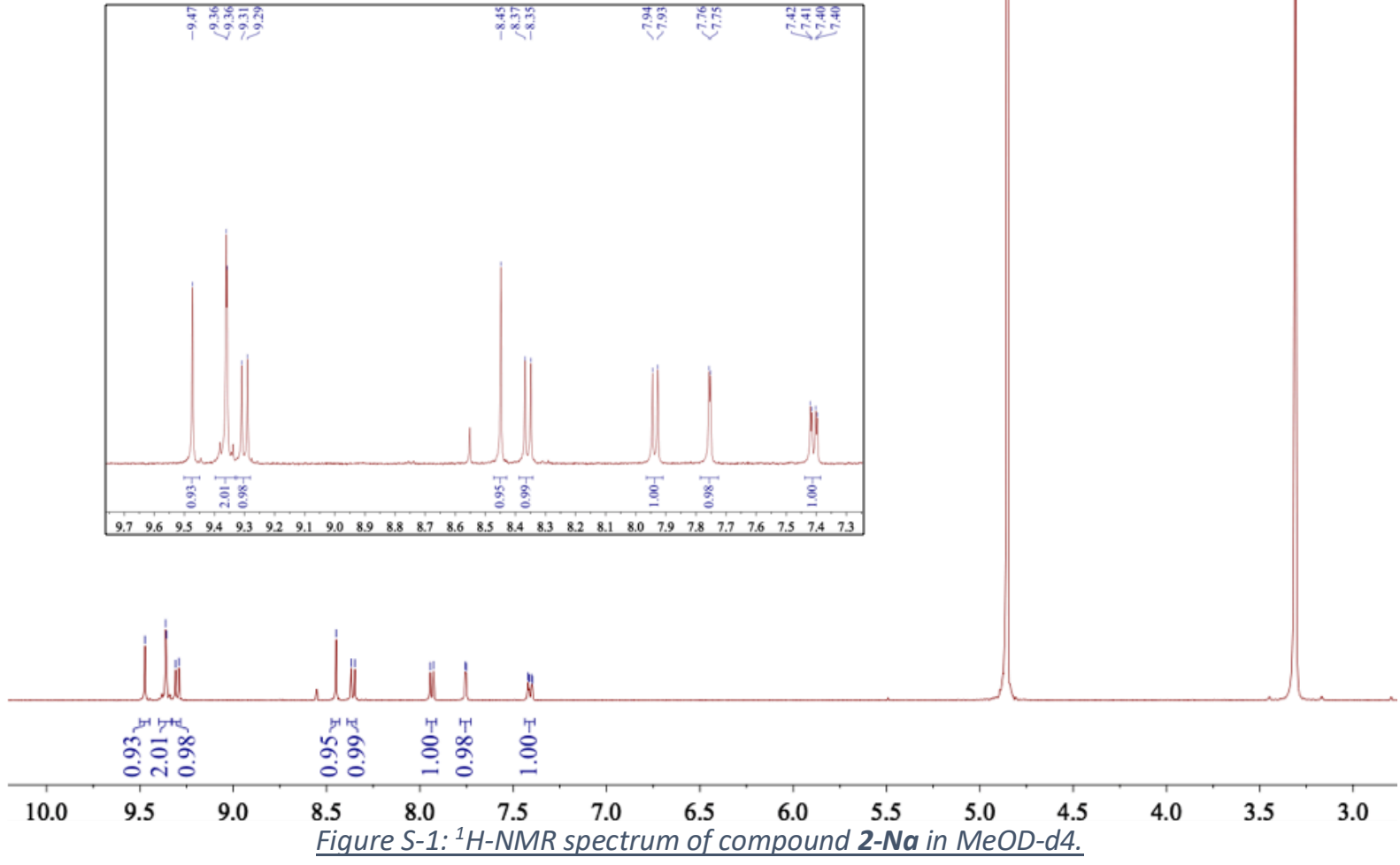

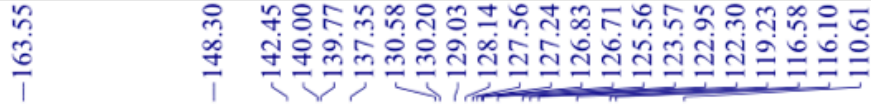

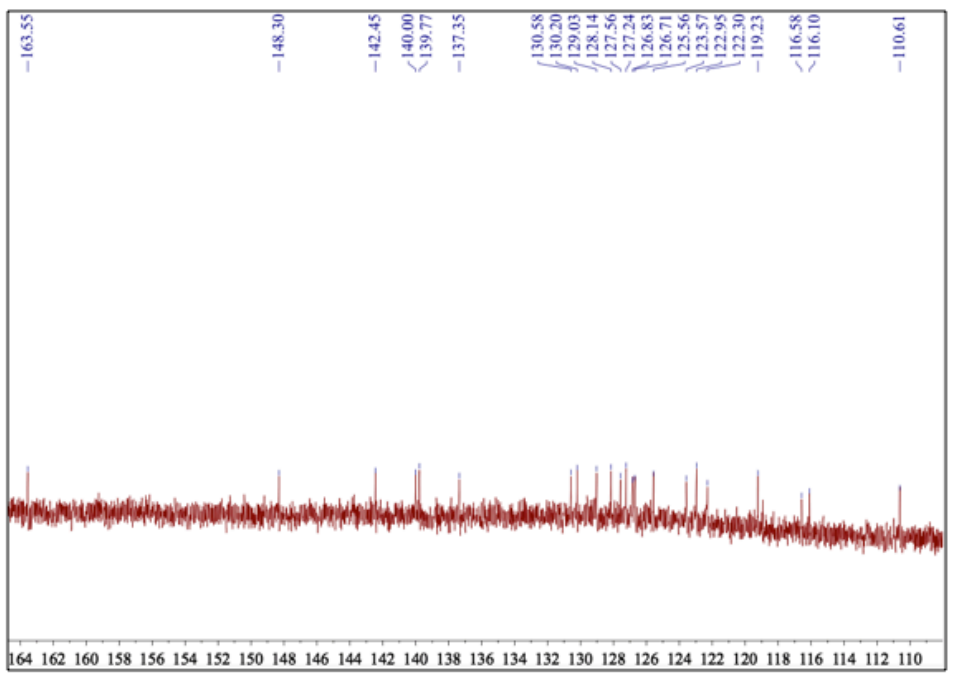

$\begin{array}{lllllllllllllllllllllllll}165 & 160 & 155 & 150 & 145 & 140 & 135 & 130 & 125 & 120 & 115 & 110 & 105 & 100 & 95 & 90 & 85 & 80 & 75 & 70 & 65 & 60 & 55 & 50 & 45\end{array}$ Figure S-2: ${ }^{13} \mathrm{C}-\mathrm{NMR}$ spectrum of compound 2-Na in MeOD-d4. 
19vl_519_neg_1 \#1-19 RT: 0.01-0.28 AV: 19 NL: 2.00E7

T: FTMS - p ESI Full ms [200.00-2000.00]
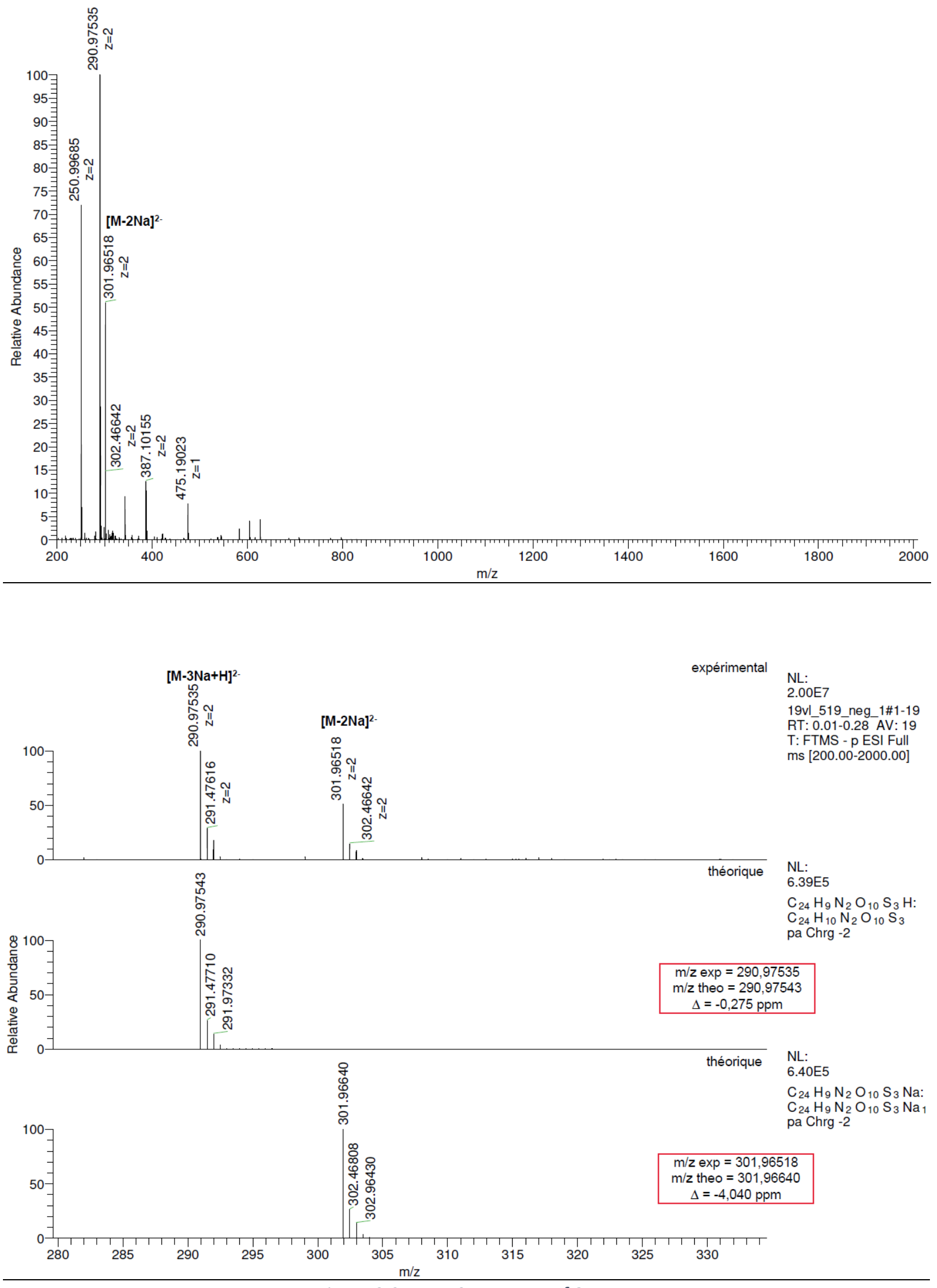

Figure S-3 : HRMS spectrum of 2-Na. 


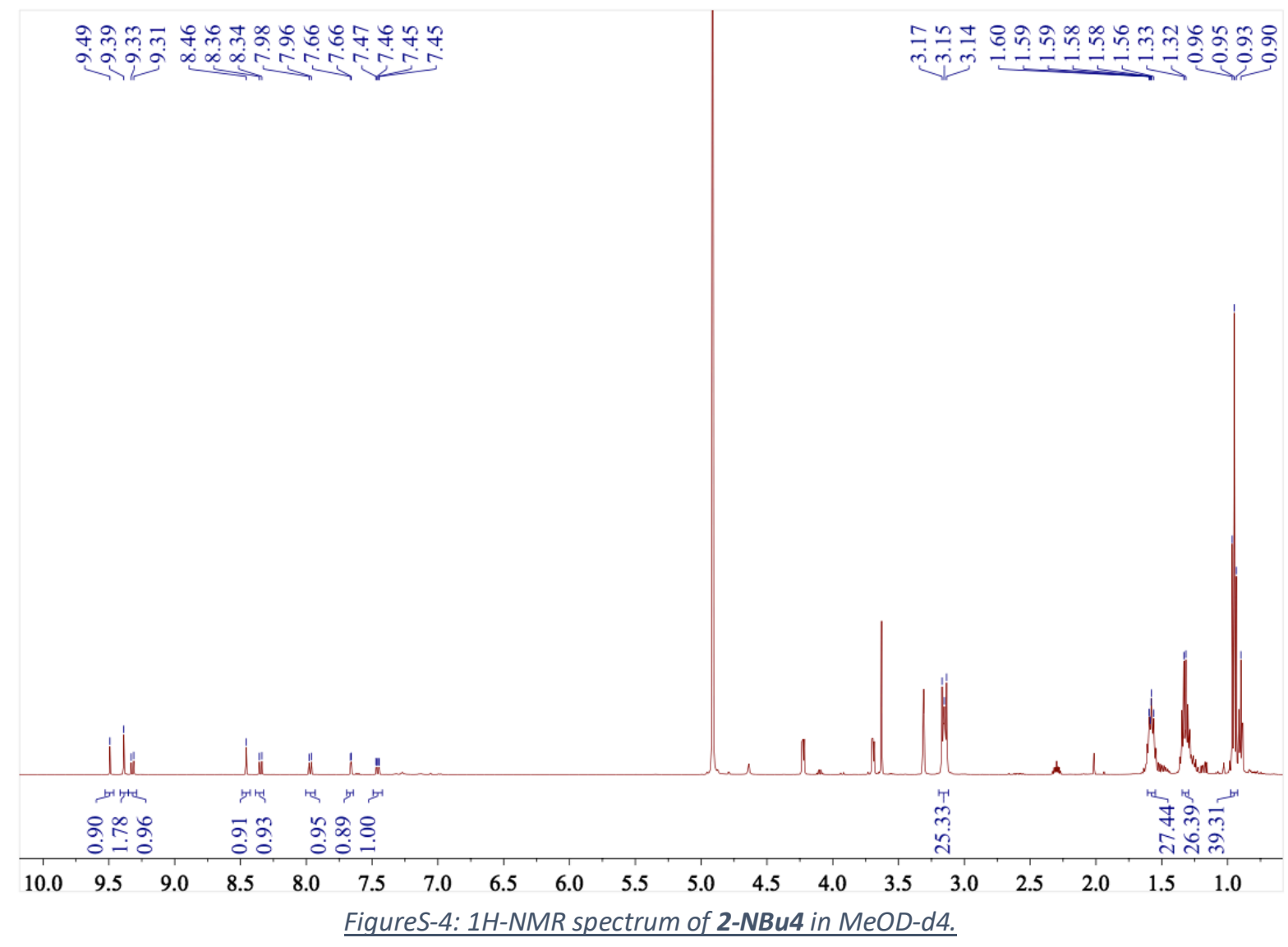

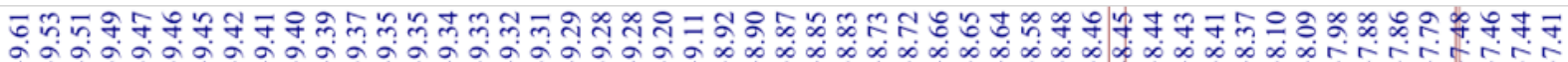
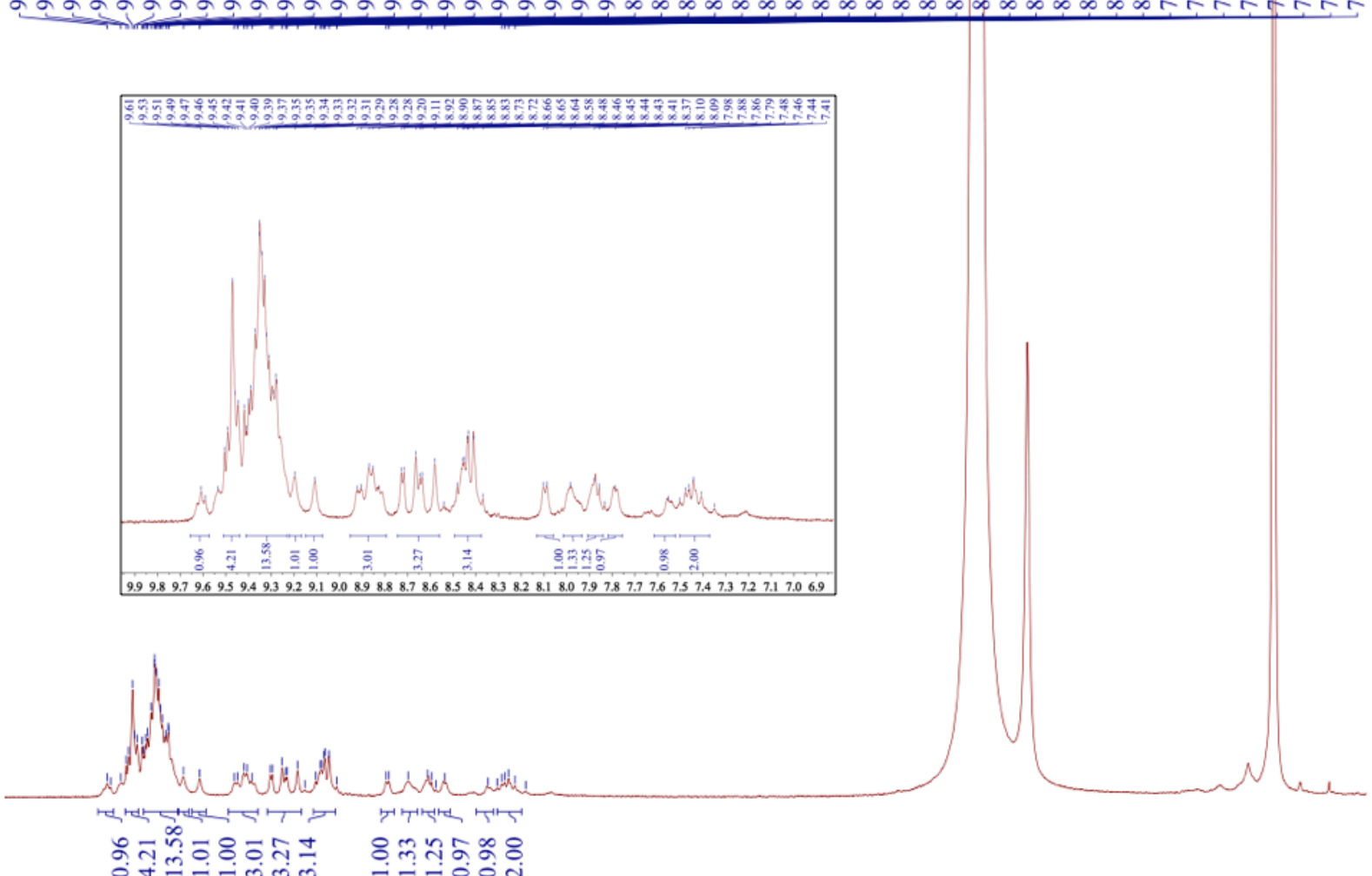

10.0

9.5

$9.0 \quad 8.5$

$8.0 \quad 7.5$

Figure S-5: ${ }^{1} \mathrm{H}-\mathrm{NMR}$ spectrum of 1-Na in MeOD-d4. 
18VI_znPcPy4neg_6 \#2-43 RT: 0.02-0.65 AV: 42 NL: 9.91E4

T: FTMS - $p$ ESI Full ms [300.00-3000.00]

mode négatif

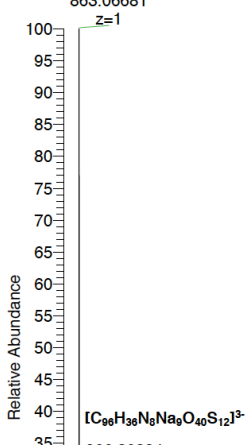

866.20234

30年 $\quad z=3$
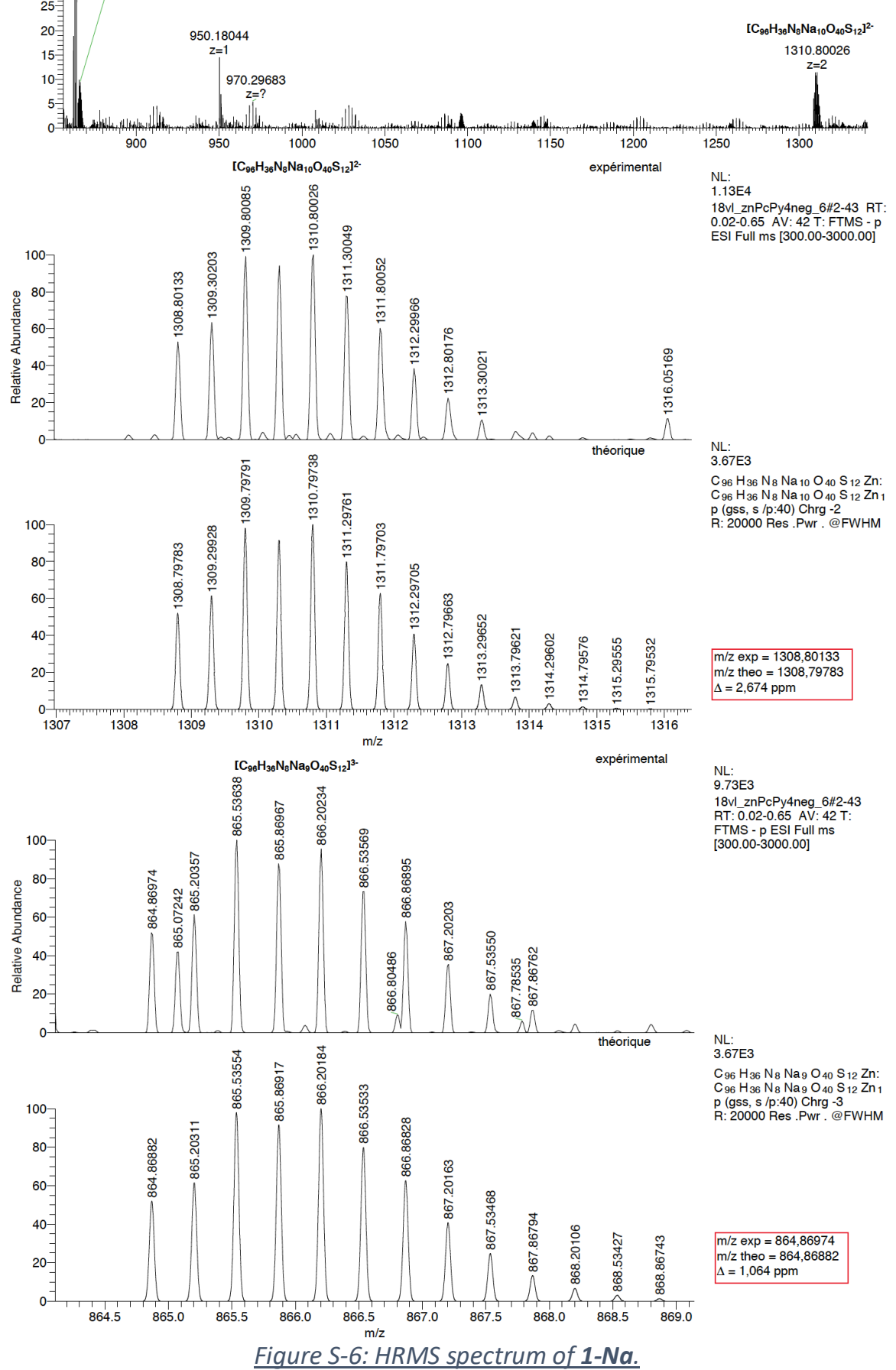


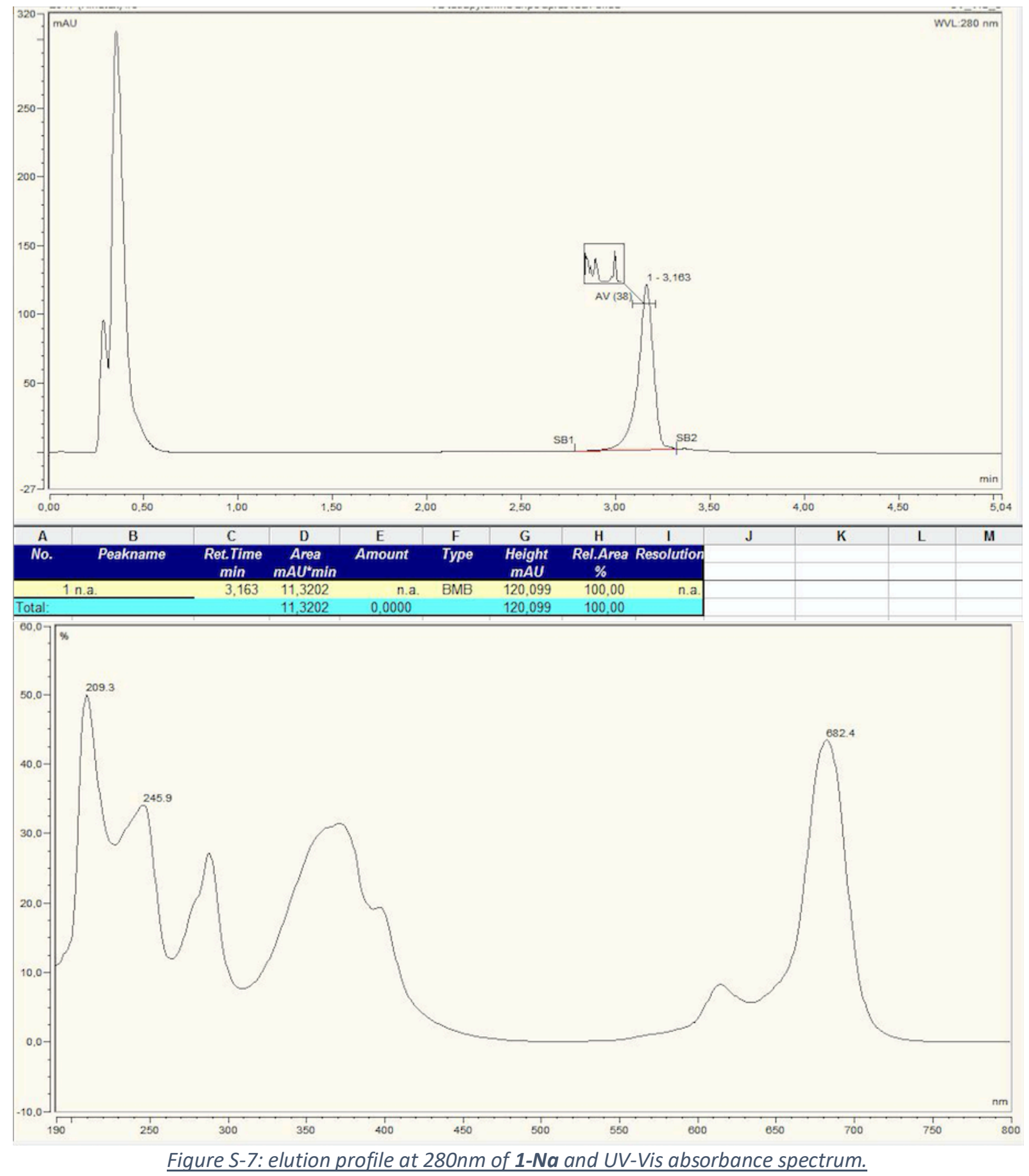




\section{Lipophilicity measurement}

To a mixture of phosphate-buffered saline (PBS) at pH $7.4(1400 \mu \mathrm{L})$ and octanol $(1500 \mu \mathrm{L})$ in a microtube was added a stock solution of 1-Na $(1 \mathrm{mM}, 100 \mu \mathrm{L})$ in PBS. The contents were vigorously mixed by vortex mixer for $20 \mathrm{~min}$ and then the phases were separated. Octanol and PBS phases were added in UV-cuvette with methanol (500 $\mu \mathrm{L}$ of octanol or PBS phase in $500 \mu \mathrm{L}$ of methanol) and UV-Vis absorbance spectrum were measured.

Numeric value of LogP was not measurable because of the total absence of product in the organic phase. 


\section{2- Cherenkov Luminescence Experiments}

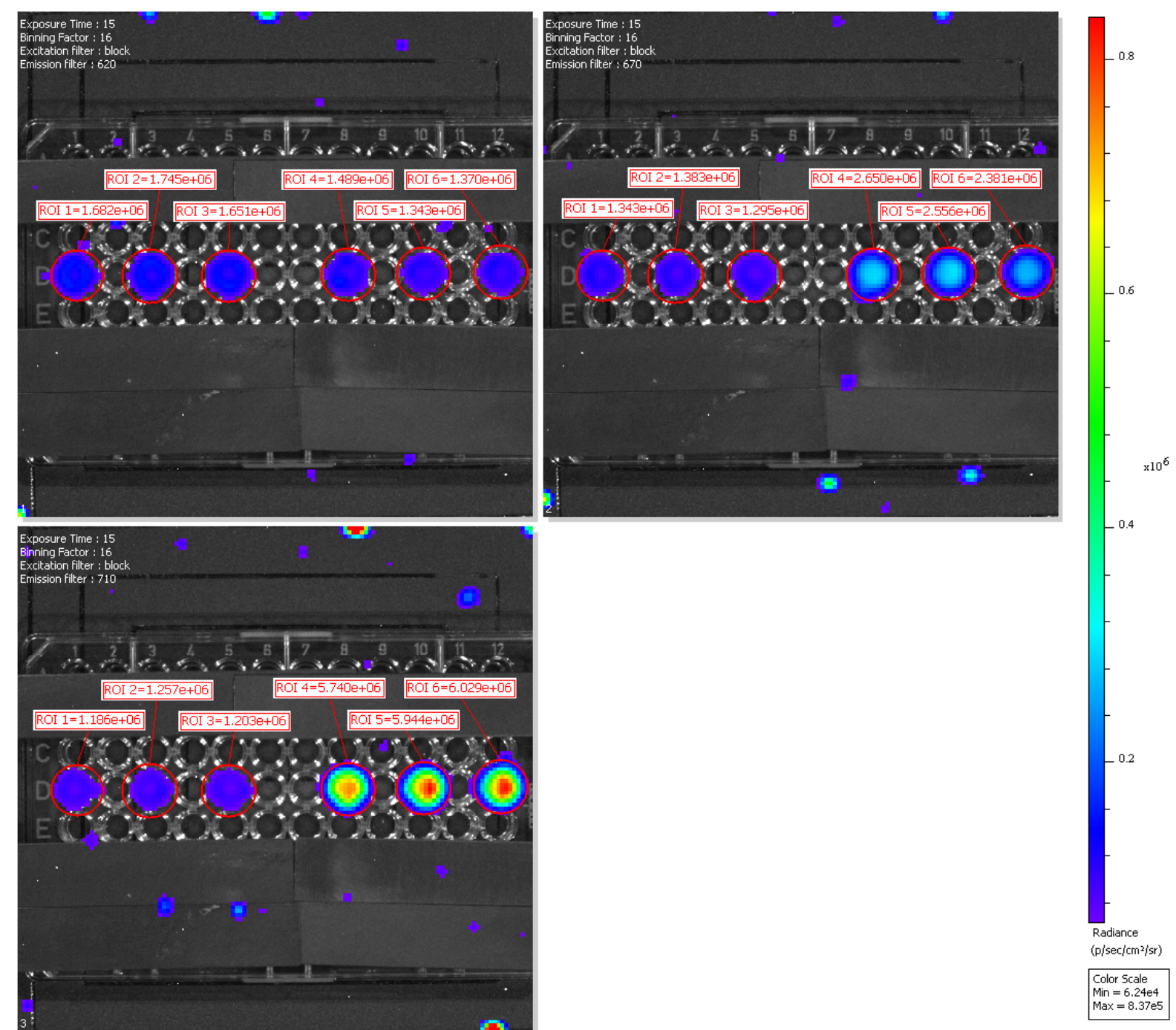

Figure S-8: radiance of ${ }^{18} \mathrm{FDG}$ alone (left) and 18FDG+1-Na (right) at 610 (up left), 670 (up right) and $710 \mathrm{~nm}$ (down left). 


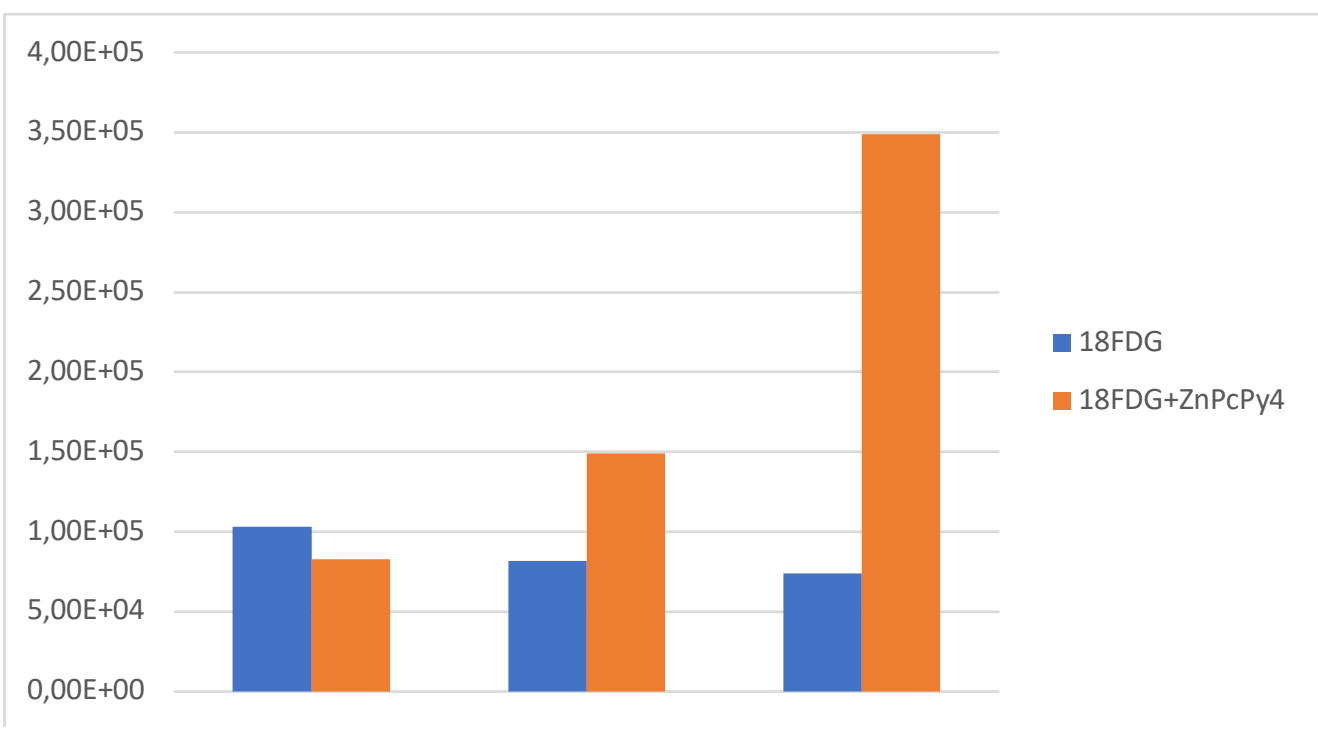

Fiqure S-9: Average radiance of ${ }^{18} \mathrm{FDG}$ alone (blue) and ${ }^{18} \mathrm{FDG}+1-\mathrm{Na}$ (red) at 610, 670 and $710 \mathrm{~nm}$.

ROI Image Layer Total Flux $[\mathrm{p} / \mathrm{s}]$ Avg Rad $\left[\mathrm{p} / \mathrm{s} / \mathrm{cm}^{2} / \mathrm{sr}\right]$ Stdev Radiance Min Radiance Max Radiance

\begin{tabular}{|c|c|c|c|c|c|c|c|c|}
\hline |VL20181115155031_001 & ROI 1 & Overlay & $1,68 \mathrm{E}+06$ & $1,09 E+05$ & $3,68 \mathrm{E}+04$ & $3,17 \mathrm{E}+04$ & $1,59 \mathrm{E}+05$ & \\
\hline VL20181115155031_001 & $\mathrm{ROI} 2$ & Overlay & $1,75 \mathrm{E}+06$ & $9,84 E+04$ & $3,84 \mathrm{E}+04$ & $3,20 E+04$ & $1,58 \mathrm{E}+05$ & $1,03 E+05$ \\
\hline VL20181115155031_001 & $\mathrm{ROI} 3$ & Overlay & $1,65 \mathrm{E}+06$ & $1,02 \mathrm{E}+05$ & $3,54 \mathrm{E}+04$ & $4,03 E+04$ & $1,57 \mathrm{E}+05$ & \\
\hline VL20181115155031_001 & $\mathrm{ROI} 4$ & Overlay & $1,49 E+06$ & $8,81 E+04$ & $3,17 E+04$ & $3,41 E+04$ & $1,54 E+05$ & \multirow{3}{*}{$8,27 \mathrm{E}+04$} \\
\hline VL20181115155031_001 & ROI 5 & Overlay & $1,34 \mathrm{E}+06$ & $8,00 E+04$ & $2,70 \mathrm{E}+04$ & $3,40 \mathrm{E}+04$ & $1,24 \mathrm{E}+05$ & \\
\hline VL20181115155031_001 & ROI 6 & Overlay & $1,37 \mathrm{E}+06$ & $7,99 \mathrm{E}+04$ & $2,93 \mathrm{E}+04$ & $2,70 \mathrm{E}+04$ & $1,30 \mathrm{E}+05$ & \\
\hline VL20181115155031_002 & ROI 1 & Overlay & $1,34 \mathrm{E}+06$ & $8,68 E+04$ & $2,81 E+04$ & $2,91 \mathrm{E}+04$ & $1,32 \mathrm{E}+05$ & \multirow{3}{*}{$8,16 \mathrm{E}+04$} \\
\hline VL20181115155031_002 & $\mathrm{ROI} 2$ & Overlay & $1,38 E+06$ & $7,80 E+04$ & $2,80 E+04$ & $3,35 \mathrm{E}+04$ & $1,20 E+05$ & \\
\hline VL20181115155031_002 & $\mathrm{ROI} 3$ & Overlay & $1,30 E+06$ & $8,01 E+04$ & $2,41 \mathrm{E}+04$ & $3,49 \mathrm{E}+04$ & $1,22 \mathrm{E}+05$ & \\
\hline VL20181115155031_002 & $\mathrm{ROI} 4$ & Overlay & $2,65 \mathrm{E}+06$ & $1,57 E+05$ & $8,12 \mathrm{E}+04$ & $3,56 \mathrm{E}+04$ & $2,92 \mathrm{E}+05$ & \multirow{3}{*}{$1,49 E+05$} \\
\hline VL20181115155031_002 & ROI 5 & Overlay & $2,56 E+06$ & $1,52 \mathrm{E}+05$ & $8,20 E+04$ & $4,13 E+04$ & $3,00 E+05$ & \\
\hline VL20181115155031_002 & ROI 6 & Overlay & $2,38 \mathrm{E}+06$ & $1,39 \mathrm{E}+05$ & $7,34 \mathrm{E}+04$ & $3,68 \mathrm{E}+04$ & $2,67 \mathrm{E}+05$ & \\
\hline VL20181115155031_003 & ROI 1 & Overlay & $1,19 \mathrm{E}+06$ & $7,67 E+04$ & $2,17 \mathrm{E}+04$ & $2,84 \mathrm{E}+04$ & $1,07 \mathrm{E}+05$ & \multirow{3}{*}{$7,40 E+04$} \\
\hline VL20181115155031_003 & $\mathrm{ROI} 2$ & Overlay & $1,26 E+06$ & $7,09 E+04$ & $2,53 \mathrm{E}+04$ & $2,98 \mathrm{E}+04$ & $1,18 \mathrm{E}+05$ & \\
\hline VL20181115155031_003 & $\mathrm{ROI} 3$ & Overlay & $1,20 \mathrm{E}+06$ & $7,43 E+04$ & $2,07 \mathrm{E}+04$ & $3,49 \mathrm{E}+04$ & $1,09 \mathrm{E}+05$ & \\
\hline VL20181115155031_003 & $\mathrm{ROI} 4$ & Overlay & $5,74 \mathrm{E}+06$ & $3,40 E+05$ & $2,22 \mathrm{E}+05$ & $5,39 E+04$ & $7,46 \mathrm{E}+05$ & \multirow{3}{*}{$3,49 E+05$} \\
\hline VL20181115155031_003 & ROI 5 & Overlay & $5,94 \mathrm{E}+06$ & $3,54 \mathrm{E}+05$ & $2,33 E+05$ & $5,88 \mathrm{E}+04$ & $8,37 E+05$ & \\
\hline VL20181115155031_003 & ROI 6 & Overlay & $6,03 E+06$ & $3,52 \mathrm{E}+05$ & $2,39 \mathrm{E}+05$ & $4,84 \mathrm{E}+04$ & $8,27 \mathrm{E}+05$ & \\
\hline
\end{tabular}

Figure S-10: ROI table for transfer experiment.

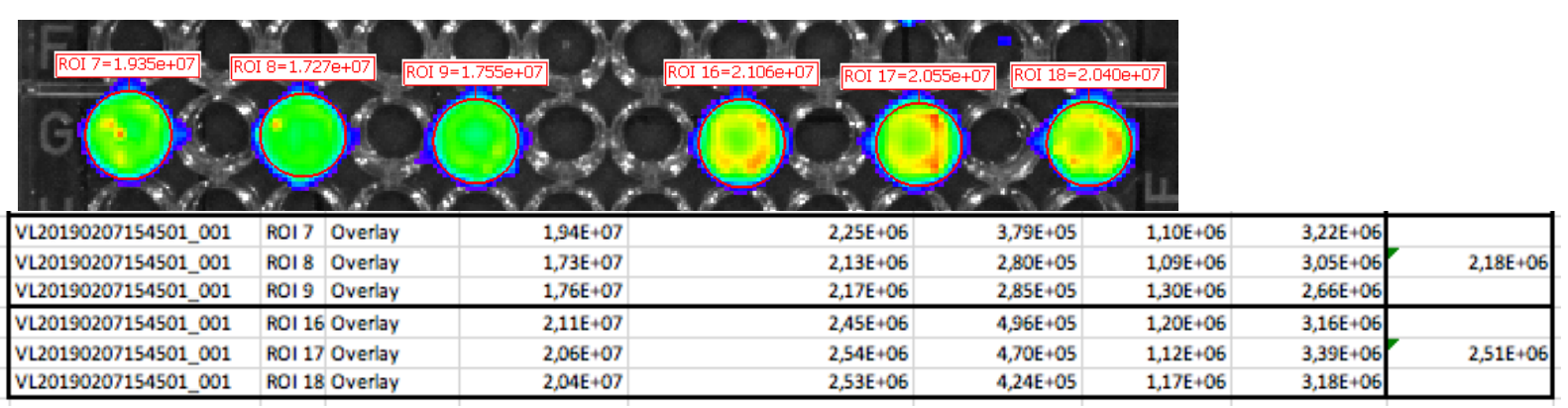

Figure S-11: ROI table in open filter. 

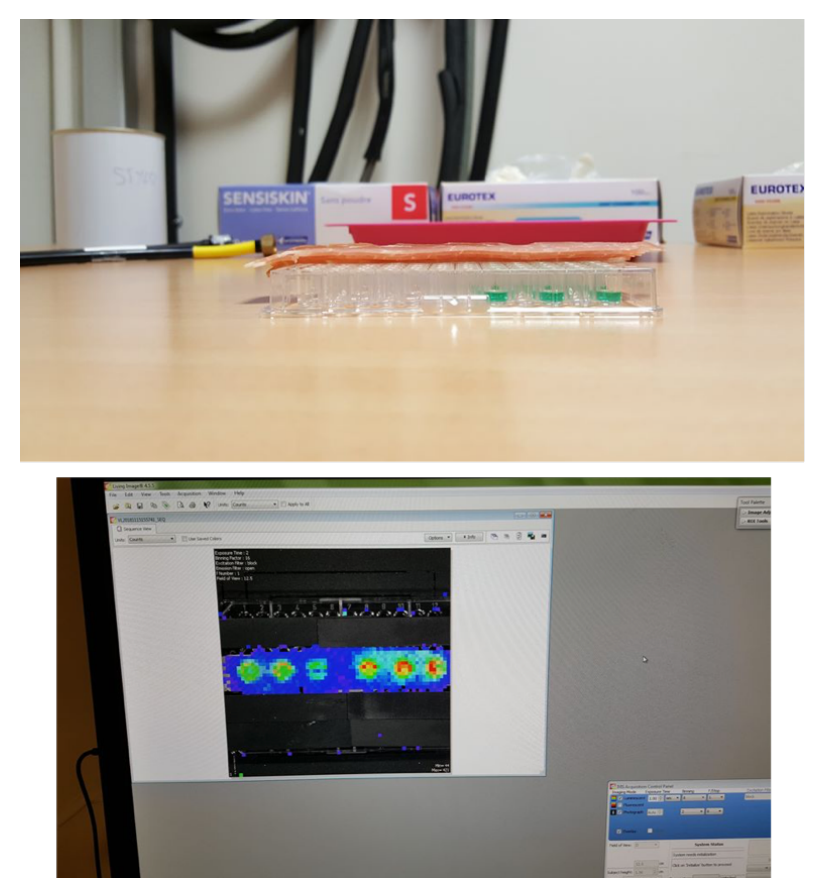

Figure S-12: Experimental Setup to achieve CLI on wells with ${ }^{18}$ F]-FDG as a CR source and upon layering slices of $\underline{\text { biological tissues. }}$

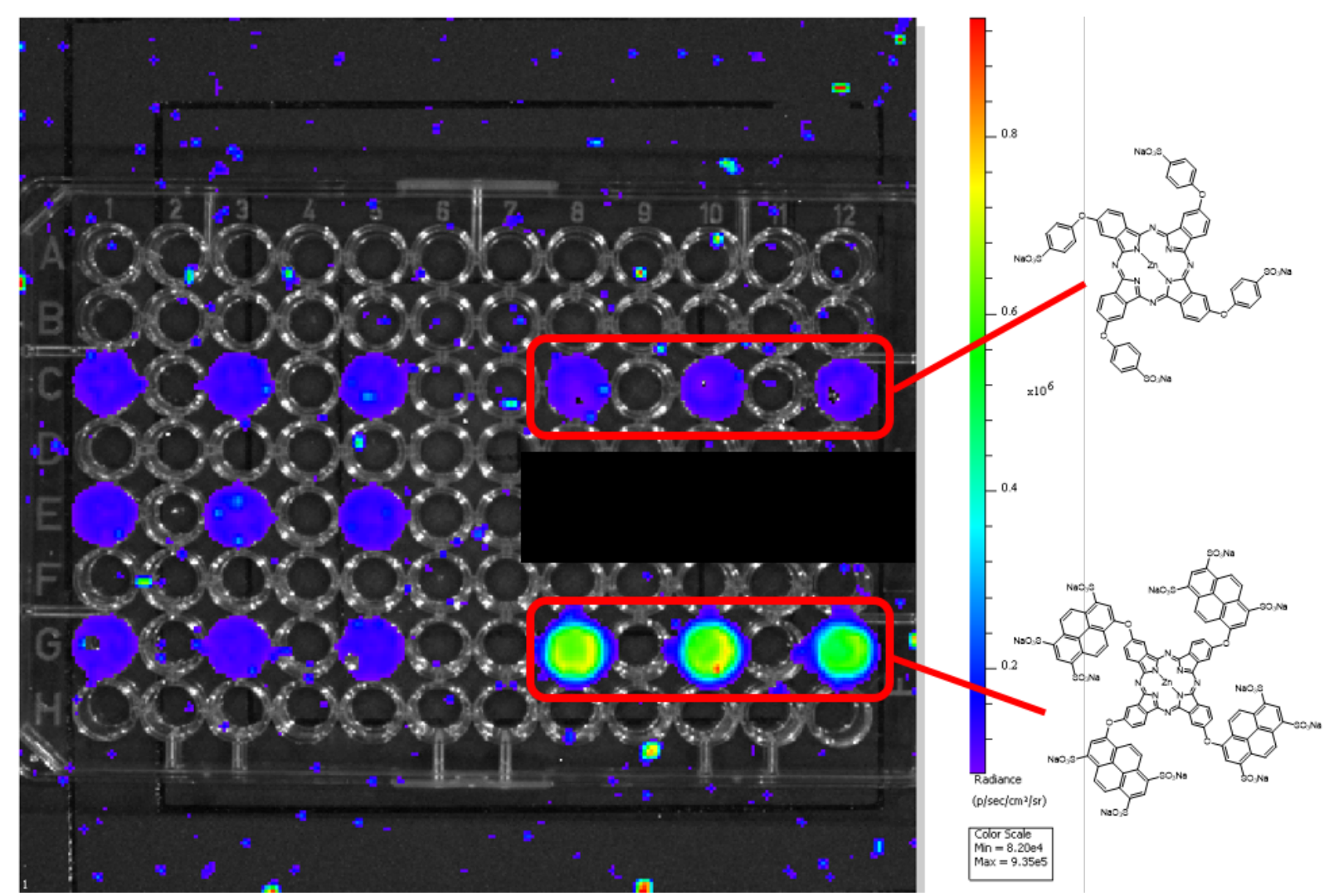

Figure S-13: radiance of ${ }^{18} \mathrm{FDG}$ alone (left), 18FDG+control PC (top right) and 18FDG+1-Na (bottom right) at 710nm. 


\begin{tabular}{|c|c|c|c|c|c|c|c|c|}
\hline Image Number & $\mathrm{ROI}$ & Image Layer & Total Flux $[\mathrm{p} / \mathrm{s}]$ & Avg Radiance $\left[\mathrm{p} / \mathrm{s} / \mathrm{cm}^{2} / \mathrm{sr}\right]$ & Stdev Radiance & Min Radiance & Max Radiance & \\
\hline VL20190207155108_003 & ROI 1 & Overlay & $9,84 E+05$ & $1,00 E+05$ & $2,25 E+04$ & $4,94 \mathrm{E}+04$ & $1,40 E+05$ & \\
\hline VL20190207155108_003 & $\mathrm{RO} 2$ & Overlay & $1,15 E+06$ & $9,05 \mathrm{E}+04$ & $2,45 E+04$ & $4,48 \mathrm{E}+04$ & $1,26 E+05$ & \\
\hline VL20190207155108_003 & ROI 3 & Overlay & $5,58 \mathrm{E}+05$ & $5,07 E+04$ & $5,64 E+05$ & $3,23 \mathrm{E}+06$ & $1,28 \mathrm{E}+05$ & \\
\hline VL20190207155108_003 & ROI 34 & 4 Overlay & $1,04 E+06$ & $9,72 \mathrm{E}+04$ & $2,82 \mathrm{E}+04$ & $3,47 E+04$ & $1,86 \mathrm{E}+05$ & \\
\hline VL20190207155108_003 & ROI 35 & 5 Overlay & $1,14 E+06$ & $8,60 \mathrm{E}+04$ & $2,73 E+04$ & $3,18 \mathrm{E}+04$ & $1,31 E+05$ & $8,99 \mathrm{E}+04$ \\
\hline VL20190207155108_003 & $\mathrm{ROI} 36$ & 6 Overlay & $1,08 E+06$ & $9,16 E+04$ & $2,46 \mathrm{E}+04$ & $4,22 \mathrm{E}+04$ & $1,33 E+05$ & \\
\hline VL20190207155108_003 & ROI 37 & Overlay & $8,78 E+05$ & $1,00 \mathrm{E}+05$ & $1,87 \mathrm{E}+04$ & $5,35 E+04$ & $1,29 \mathrm{E}+05$ & \\
\hline VL20190207155108_003 & $\mathrm{RO} 38$ & 8 Overlay & $9,54 \mathrm{E}+05$ & $9,47 E+04$ & $2,03 E+04$ & $5,13 E+04$ & $1,22 E+05$ & \\
\hline VL20190207155108_003 & ROI 39 & Overlay & $9,41 \mathrm{E}+05$ & $9,82 \mathrm{E}+04$ & $2,06 E+04$ & $5,18 \mathrm{E}+04$ & $1,27 E+05$ & \\
\hline VL20190207155108_003 & $\mathrm{ROI} 40$ & Overlay & $8,92 \mathrm{E}+05$ & $9,19 E+04$ & $1,49 \mathrm{E}+04$ & $5,70 E+04$ & $1,15 \mathrm{E}+05$ & \\
\hline VL20190207155108_003 & ROI 41 & 1 Overlay & $8,46 \mathrm{E}+05$ & $9,54 \mathrm{E}+04$ & $1,51 E+04$ & $5,68 E+04$ & $1,16 E+05$ & $9,21 E+04$ \\
\hline VL20190207155108_003 & $\mathrm{ROOI} 42$ & 2 Overlay & $7,79 \mathrm{E}+05$ & $8,91 E+04$ & $1,96 \mathrm{E}+04$ & $2,76 E+04$ & $1,17 E+05$ & \\
\hline
\end{tabular}

Figure S-14: ROI table for antenna control experiment. 


\section{3- ROS generation}
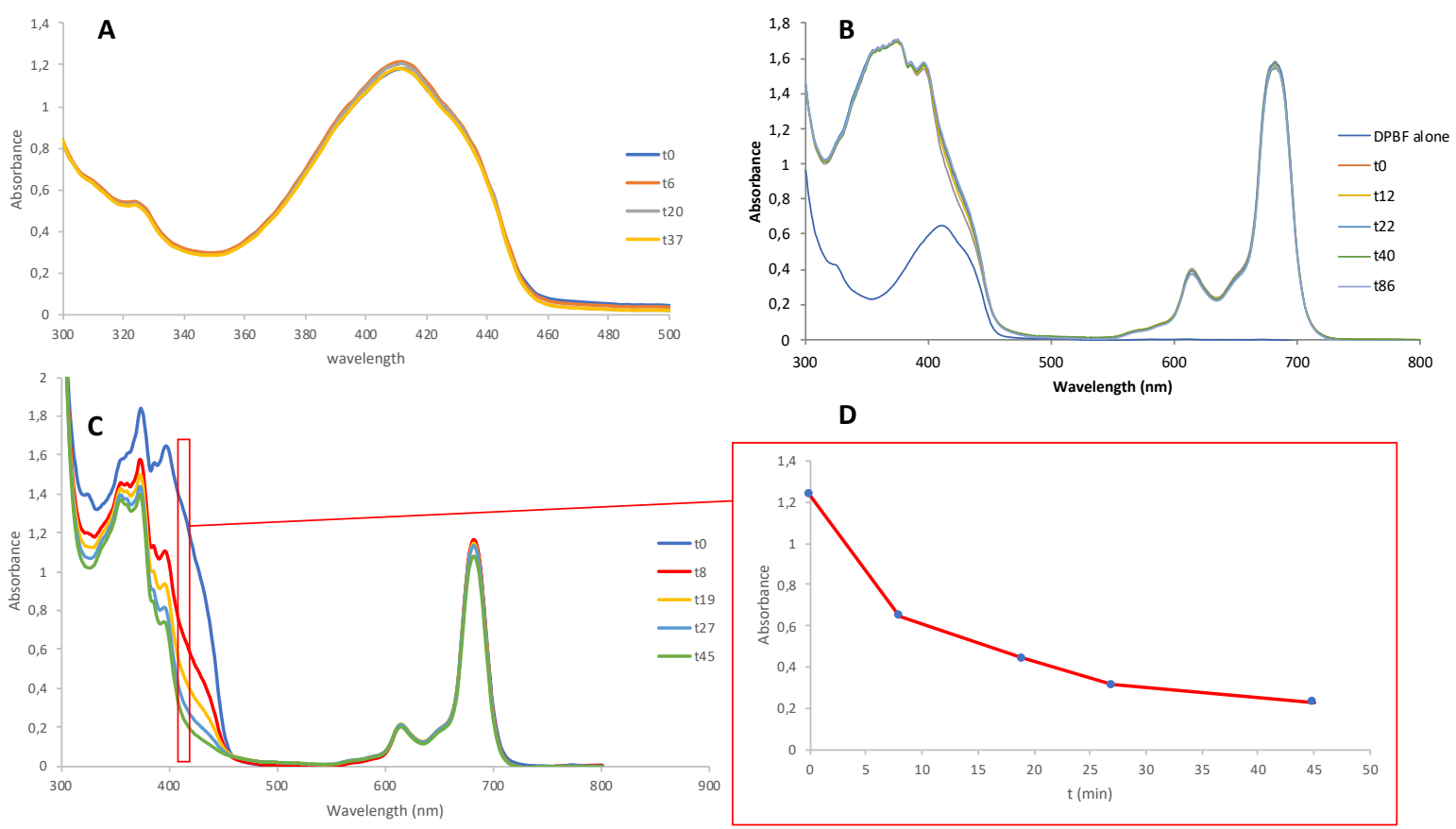

Figure S-15: UV/Vis spectrum of DPBF alone (A), DPBF and $Z n P c P y_{4} 1$ without ambient light (B), Reaction of $D P B F$ and $R O S$ generated upon irradiation of $Z n P C P y_{4} 1$ with ambient light $(C \& D)$ 